MEMÓRIA

\title{
O Instituto de Tecnologia Industrial de Minas Gerais
}

João Antonio de Paula*

O Instituto de Tecnologia Industrial de Minas Gerais, criado em 1944, como órgão da Secretaria de Agricultura, Indústria, Comércio e Trabalho do Estado de Minas Gerais, foi inspirado tanto no Instituto de Pesquisas Tecnológicas, de São Paulo, implantado em 1927, quanto no Instituto Nacional de Tecnologia, que funcionava no Rio de Janeiro. O ITI de Minas Gerais fez parte de um conjunto de ações desenvolvidas pela Secretaria da Agricultura que, naquela época, tinha a seu cargo uma série de atribuições que configuravam o que se chama hoje de "desenvolvimentistas". Com efeito, desde o final do século XIX, como resultado das repercussões em Minas Gerais da implantação da República, o Estado vai experimentar uma série de transformações econômicas, políticas, sociais e culturais, informadas por dois grandes vetores: a busca da modernização econômica e institucional; e a afirmação do Estado como sujeito importante do processo de desenvolvimento regional e nacional.

Esses dois vetores estiveram, exemplarmente, presentes na atuação de João Pinheiro, seja durante a campanha republicana, na qual se destacou, seja em sua curta passagem pelo governo de Minas, no início do regime republicano, seja em sua atuação à frente do Congresso Agrícola, Comercial e Industrial de Minas Gerais, de 1903, que se constituiu em efetiva construção de um programa de desenvolvimento

* Professor do Centro de Desenvolvimento e Planejamento Regional da Faculdade de Ciências Econômicas (Cedeplar/Face), da Universidade Federal de Minas Gerais (UFMG), Belo Horizonte-MG, Brasil. E-mail: jpaula@cedeplar.ufmg.br 
econômico para Minas Gerais, seja, decisivamente, durante sua nova passagem pelo governo de Minas, entre 1906 e 1908.

A morte de João Pinheiro, em 1908, interrompeu uma carreira, singular entre nossos políticos, marcada pelo dinamismo e sentido republicano de sua atuação. Seu exemplo deixou frutos importantes, pois vários de seus colaboradores e aliados exerceram importantes papéis na administração pública e no Parlamento federais, a partir do governo de Afonso Pena, 1906-1909. O grupo de políticos mineiros ligados a João Pinheiro, chamado de "Jardim da Infância" e composto por nomes como João Pandiá Calógeras, João Luis Alves, David Campista, Gastão da Cunha, Carlos Peixoto, teve atuação destacada na cena política e administrativa federal, com iniciativas de cunho modernizante, que se chocaram com as velhas práticas oligárquicas e conservadores, que comandavam a política nacional.

A atuação do "Jardim da Infância" tem algo de fundacional de uma política que não será equívoco se for chamada de "desenvolvimentista" e que marcou a vida nacional, a partir da década de 1930, mas que teve antecedentes importantes nas iniciativas políticas e empresariais de Teófilo Otoni e na atuação político-administrativa de João Pinheiro.

Com efeito, consolidou-se, precocemente, em Minas Gerais, certa cultura política, que reconheceu central o papel do Estado como sujeito importante do processo de desenvolvimento econômico, político, social e cultural. Esta tradição valeu a Minas Gerais lugar de destaque na política nacional durante a República Velha, mesmo antes do acordo, em 1913, que definiu a alternância entre mineiros e paulistas na Presidência da República.

Minas Gerais dividiu com o Rio Grande do Sul e a Paraíba a liderança do movimento vitorioso em 1930. Este fato garantiu ao Estado autonomia na condução da política regional, ao contrário do que aconteceu em outros estados em que o governo federal interveio fortemente. Assim, o presidente eleito de Minas Gerais, Olegário Maciel, exerceu seu mandato até 1933, quando faleceu, sendo substituído por Benedito Valadares, que governou o estado até 1945. Tido, por certo folclore, como político atrasado, Benedito Valadares caracterizou seu longo governo por medidas modernizantes e "desenvolvimentistas": foi dele a escolha de Juscelino Kubitschek para a Prefeitura de Belo Horizonte, entre 1940 e 1945; em seu governo foi criada a Cidade Industrial, em 1941; e a Secretaria de Agricultura, tendo à frente Israel Pinheiro, entre 1934 e 1942, e posteriormente Lucas Lopes, 
entre 1943 e 1945, desenvolveu uma série de políticas e iniciativas inovadoras, como é o caso da criação do ITI.

Quando da inauguração do ITI, em 1944, o secretário de Agricultura Lucas Lopes, disse:

Este laboratório foi criado pelo Governo para se tornar um centro de irradiação de ampla e profunda cultura técnica, um núcleo de formação de engenheiros altamente especializados e capazes. Será, também, um centro de pesquisas industriais e cientificas para aqueles que já estão fabricando as nossas máquinas, os nossos produtos manufaturados. [...]

Só agora é proporcionado aos que aqui produzem, o campo fecundo de experimentação cientifica, de pesquisa sistematizada, de controle racional da produção. [...]

Não sendo uma escola de engenharia ele será, contudo, a parte viva do ensino de engenharia. Sem ser um combinado de usinas ou de oficinas, ele será, entretanto, o órgão de controle e de aperfeiçoamento de nosso parque industrial.

Mais do que isto, este Instituto, estabelecendo a articulação entre a indústria e o ensino, entre o gabinete e as oficinas, entre o laboratório e a fábrica, terá relevante e indiscutivel missão social (LOPES apud GUIMARÃES, 1990a, p. 331).

Não será ocioso sublinhar as significativas inovações e articulações institucionais pretendidas pelo ITI, que, de fato, foi experimento concreto, com expressivos resultados, na viabilização de interação entre governo, institutos de pesquisa, universidades e empresas.

Fundador do ITI, Lucas Lopes, um dos grandes homens públicos do Brasil, com atuação destacada como engenheiro, gestor de empresas, secretário de Estado, ministro e primeiro-presidente das Centrais Elétricas de Minas Gerais, declarou lamentar "os problemas burocráticos e políticos que dificultaram o funcionamento do ITI, finalmente incorporado ao Centro Tecnológico de Minas Gerais (Cetec).” (LOPES apud DINIZ, 2008, p. 83).

A criação do ITI foi o resultado de uma confluência de instituições e a confirmação de certas vocaçôes. O ITI foi implantado durante a gestão de Lucas Lopes à frente a Secretaria de Agricultura de Minas Gerais. Engenheiro formado pela Escola de Engenharia da UFMG, em 1932, Lucas Lopes teve entre seus mais importantes 
colaboradores vários nomes formados pela Escola de Minas de Ouro Preto. O ITI também será a vocação mineratória de Minas Gerais, pela decisiva importância que ali sempre tiveram a geologia, a mineralogia e a metalurgia.

O ITI de Minas Gerais, foi criado pelo Decreto-Lei n. 983, de 9 de dezembro de 1943, que organizou a Secretaria da Agricultura, Induistria, Comércio e Trabalho. Foi inaugurado em 2 de julho de 1944, com a presença do Presidente da República Getúlio Vargas, e reorganizado pelo Decreto-Lei n. 1516, de 12 de dezembro de 1945. A reorganização do instituto visou atender ao aumento e diversificação dos pedidos de assistência técnica especializada. [...] Além de um diretor com mandato de 4 anos, e de livre nomeação do governador, passou a ter um Conselho Técnico Consultivo, constituido de 6 membros, representantes das seguintes entidades: Escola de Engenharia da UMG, Secretaria de Agricultura, Federação das Indistrias, Secretaria da Viação, Sociedade Mineira de Engenheiros e Sociedade Mineira de Agricultores. [...] O ITI passaria a funcionar em regime de tempo integral com adicional de 50\% nos salários vigentes de seus servidores. Sua estrutura orgânica passou a ser a seguinte:

\section{Setores}

a. Química Tecnológica

b. Geologia de Minas

c. Metalurgia

d. Materiais de construção

e. Física Tecnológica

f. Combustíveis e Motores Térmicos

g. Estabilidade das construções

h. Indústrias Têxteis

\section{Serviços Auxiliares}

a. Seção Administrativa

I. Secretaria

II. Contabilidade

III. Almoxarifado

IX. Portaria

b. Documentos e Divulgação

I. Biblioteca

II. Arquivo e Documentação

III. Publicaçōes

IV. Serviço Fotográfico. (GUIMARÃES, 1990a, p. 391-392). 
Publicação do I.T.I.

Boletim $n^{0} 25$

ESTADO OE MINAS GERAIS

INSTITUTO DE TECNOLOGIA INDUSTRIAL

THEOPHILO DIAS PACS LEME, OIRETOR

\section{BOLETIM NO25} ac. 592397

U.F.M.G. - BIBLIOTECA UNIVERSITÁRIA

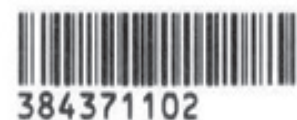

NĀO DANIFIQUE ESTA ETIQUETA

\section{HISTORRCOC E ATUACÄO OO I.T.I. NO DESENYOVINEETO TECWICOCEENTFFCO \\ EM MINAS GERAIS}

BELO HORIZONTE

1958

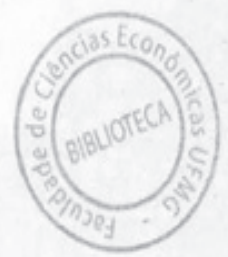


Entre os muitos aspectos denotadores de excelência e atualização do ITI, destaca-se a significativa atenção dada pela Instituição à biblioteca, ao arquivo e às publicações. Nessas áreas o ITI contou com a decisiva presença do pesquisador, bibliófilo, bibliotecário e arquivista Hélio Gavatá. Sobre ele disse Cristina Ávila (1998, p. 19):

Dedicou-se também a trabalhos de assessoramento a diversas instituições, como ao Instituto de Tecnologia Industrial, montando a Biblioteca da instituição, que mantinha em seu acervo as mais fundamentais publicaçôes, modificando a infraestrutura da pesquisa técnica em Minas Gerais, levantando as fontes mais originais sobre temas específicos, pesquisando, indicando e sugerindo a compra do que havia de mais avançado na época (ÁVILA, 1998, p. 19).

Instalado, inicialmente, em dependência da Escola de Engenharia da UFMG, o ITI passou a funcionar no Instituto de Química da Escola de Engenharia da UFMG, na Rua da Bahia n. 52:

Juntamente com os químicos, vários engenheiros de Minas (a maioria deles formados em Ouro Preto) organizaram o que ficou conhecido como o "Laboratório da Rua da Bahia 52". Esse grupo, que incluia Djalma Guimarães, Otávio Barbosa, Sebastiāo Virgílio Ferreira, Olinto Vieira Pereira e Manuel Pimentel Godói, foi responsável pela criação do Instituto de Tecnologia Industrial de Minas Gerais, fundado em 1944 e mais tarde transformado no Centro de Tecnologia do Estado (SCHWARTZMAN, 2015, p. 184).

O ensino e a pesquisa de Química na UFMG, tanto na Faculdade de Medicina, quanto na Escola de Engenharia, foram profundamente marcados pela atuação do professor Alfred Schaeffer, formado em Química pela Universidade de Munique, que veio para Belo Horizonte para trabalhar no Instituto Filial de Manguinhos, instalado na cidade em 1907. Depois de montar o setor de Química do Instituto, Alfred Schaeffer foi nome fundamental na instauração da cultura científica e tecnológica de primeira linha em Belo Horizonte, seja na Faculdade de Medicina, seja na Escola de Química.

O ITI expandiu suas atividades ao longo da década de 1950, com oscilações algo erráticas, sendo que a partir de 1958 as atividades do Instituto foram significativamente diminuídas. 
TABELA 1

Atividades desenvolvidas pelo ITI - 1946-1955

\begin{tabular}{cccc}
\hline Anos & Certificados & Amostras & Ensaios \\
\hline 1946 & 1.072 & 2.661 & 1.733 \\
1947 & 1.247 & 3.501 & 8.536 \\
1948 & 1.525 & 3.125 & 5.740 \\
1949 & 2.762 & 3.706 & 12.247 \\
- & - & - & - \\
1951 & 3.258 & 3.869 & 14.140 \\
1952 & 9.930 & 6.580 & 12.800 \\
1953 & 1.296 & 1.716 & 5.263 \\
1954 & 5.057 & 1.558 & 4.255 \\
1955 & 1.025 & 12.159 & 4.661 \\
\hline
\end{tabular}

Fonte: Guimarães (1990b).

Para chefiar o setor de geologia e geoquímica do ITI, Lucas Lopes convidou o renomado geólogo Djalma Guimarães, formado pela Escola de Minas de Ouro Preto, que encontrou os laboratórios do ITI já estruturados por um grupo de químicos do antigo Serviço de Produção Mineral, órgão federal. Entre eles havia dois alemães, Alfred Schaeffer e Otto Rothe, esse último tendo se tornado posteriormente professor de Química Orgânica na Faculdade Nacional de Filosofia, no Rio de Janeiro. Durante o governo de Juscelino Kubitschek em Minas Gerais, o órgão foi dirigido pelo engenheiro José Moreira dos Santos Pena. Lamentavelmente, no governo Bias Fortes o ITI entrou em decomposição e foi fechado (PEREIRA, 2015, p. 73-74).

A Escola de Minas de Ouro Preto, instalada em 1876, é outra instituição protagonista do desenvolvimento científico e tecnológico no Brasil em áreas como a geologia, a mineralogia e a metalurgia. Iniciativa do Imperador Pedro II, a Escola de Minas de Ouro Preto teve como seu primeiro diretor Claude Henri Gorceix, licenciado em ciências físicas e matemáticas, em 1866, pela École Normale Supérieure, de Paris, que foi nomeado "agregé-preparateur" de geologia e mineralogia na École Normale. Depois de período de estudos na Grécia e de ter participado da Guerra Franco-Prussiana, entre 1870 e 1871, ele "publicou várias memórias nos Anais da École Normale e nos Anais de Química e Física de Paris. Foi então que recebeu o convite para vir ao Brasil e o aceitou" (CARVALHO, 1978, p. 25-26).

A Escola de Minas de Ouro Preto, como o Instituto Oswaldo Cruz, foi uma “instituição de instituições”. Sua importância na constituição do Serviço Geológico e 
Mineralógico do Brasil, em 1907, foi central, assim como sua participação na criação da Escola de Engenharia da Universidade Federal de Minas Gerais, em 1911, uma vez que vários dos fundadores dessas duas instituiçôes foram formados pela Escola de Minas, no espírito de Gorceix, no espírito do ensino e da pesquisa, na pesquisa fundada na metodologia positivista.

O papel da Escola de Minas de Ouro Preto no desenvolvimento científico e tecnológico brasileiro foi destacado por observadores insuspeitos

Um trabalho, insuspeito por ter sido escrito por ex-aluno da Escola Politécnica, velha rival da Escola de Minas, é o de Othon H. Leonardos. Entre os nomes que Leonardos julga importante mencionar na mineralogia até mais ou menos 1940, a grande maioria é constituida de ex-alunos de Ouro Preto. O que Leonardos chama o "grupo moderno do Rio de Janeiro", que girou principalmente em torno do SGMB e do DNPM, é composto por 19 ex-alunos e de apenas 16 formados no exterior ou em outras escolas brasileiras. O que chama de "grupo de Minas", organizado em torno do Instituto de Tecnologia Industrial e da Divisäo de Fomento Mineral, sob a liderança de Djalma Guimarães, que se compóe quase que totalmente de ex-alunos. Na formação do novo grupo de São Paulo há a contribuição de elementos de Ouro Preto, como L. F. Morais Rego, Otávio Barbosa, Alceu Barbosa. O grupo que trabalha no Rio Grande do Sul é todo ele de ex-alunos. [...] Ponto importante a notar é que, aparentemente, a maior produção da pesquisa dos ex-alunos foi feita fora da Escola após a criação do SGMB. Neste órgão, no DNPM, no ITI de Minas Gerais e em outros órgãos do governo, parecem terem se refugiado os melhores pesquisadores para exercer seu trabalho. [...] [entre esses] Djalma Guimarães, talvez a maior vocação de cientista produzida na Escola (CARVALHO, 1978, p. 92-94-95).

Região mineratória por antonomásia, Minas Gerais foi objeto de permanente atenção por parte de naturalistas, geólogos e engenheiros de minas, geoquímicos, desde o período colonial. A fundação da Escola de Minas de Ouro Preto consolidou uma longa tradição de estudos e pesquisas, dando-lhe sólidos fundamentos científicos e formando gerações de engenheiros e geólogos, que vão criar o Serviço Geológico e Mineralógico do Brasil e seus desdobramentos regionais. Entre os grandes nomes formados pela Escola de Minas de Ouro Perto, um merece destaque especial, pela excelência de seu trabalho, que o coloca entre os grandes cientistas brasileiros: Djalma Guimarães. Nascido em 1894, em Santa Luzia, Minas Gerais, e falecido em 1973, 
em Belo Horizonte, Djalma Guimarães tem biografia que se confunde com aspectos importantes da história de Minas Gerais. Pertence à família Guimarães, que deu a Minas Gerais e ao Brasil escritores importantes, como Bernardo de Guimarães, Alphonsus de Guimaraens, João Alphonsus e Alphonsus de Guimarães Filho.

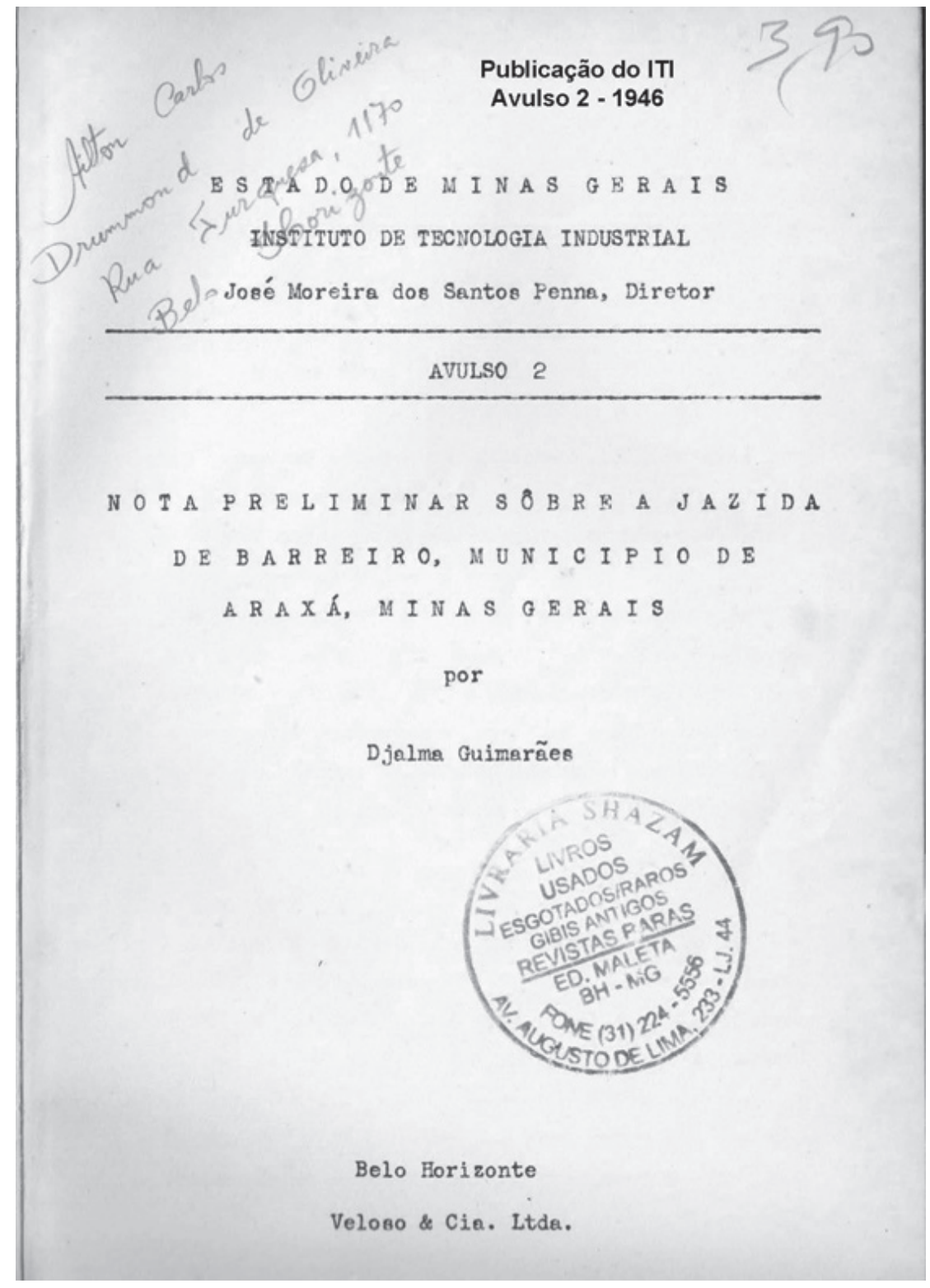


Djalma Guimarães era sobrinho de João Pandiá Calógeras, que, por sua vez, era genro do irmão de Bernardo Guimarães, Joaquim Caetano da Silva Guimarães, ministro do Supremo Tribunal de Justiça. Convivendo desde cedo com Pandiá Calógeras, terá sido esta a principal influência para que ingressasse na Escola de Minas de Ouro Preto, onde seu tio havia se formado, em 1890, concluindo o curso com brilhantismo, em 1919. Por seu desempenho, Djalma foi premiado com viagem à Europa, a qual recusou iniciando logo sua carreira profissional na Estrada de Ferro de Teresópolis. Mas, foi com pesquisas nos campos da geologia, mineralogia, petrologia e geoquímica que se notabilizou. Logo contratado pelo Serviço Geológico e Mineralógico do Brasil, já no início dos anos 1920, iniciou uma notável carreira como pesquisador, publicando, em 1924, Contribuição à petrographia do Brasil (GUIMARÃES, 1924). Sua fama logo se estabeleceu. Na biografia de Calógeras, Antônio Gontijo de Carvalho (1935, p. 57) diz: "Djalma Guimarães, seu sobrinho é hoje o maior petrographo brasileiro.” Seu prestígio transcendeu o país, destacando-se por suas publicações e participações em congressos internacionais. Publicou mais de 240 trabalhos científicos, alguns com impacto internacional, como Origem dos granitos - Das problem der granitbildung, publicado no $1^{\circ}$ Congresso de Geologia, em Nuremberg, em 1938. Djalma Guimarães foi membro do Conselho Nacional de Pesquisa, da Academia Brasileira de Ciências, da American Society of Geology, da Societé Geologique de France, da Sociedade Brasileira de Geologia, da Comission Geochemique, de La Union International de Chemie Pure e Apliquée, de Geological Society of America (New York) e da American Geophysicial Union (Washington), além de editor associado da Economic Geology.

Foi professor em três universidades brasileiras: Escola de Minas de Ouro Preto, Universidade do Distrito Federal e Universidade Federal de Minas Gerais. Dirigiu o Departamento Nacional de Produção Mineral (DNPM), a Companhia de Pesquisa de Recursos Minerais (CPRM), o Serviço Geológico do Brasil e o Instituto de Tecnologia Industrial de Minas Gerais (ITI). Foi um dos fundadores, em 1952, do Instituto de Pesquisas Radioativas (IPR), da Escola de Engenharia da Universidade Federal de Minas Gerais (UFMG), que é hoje o Centro de Desenvolvimento de Tecnologia Nuclear (CDTN).

Descreveu quatro novos minerais, os quais denominou com grandes nomes da geologia brasileira: eschwegeita, em homenagem a Ludwig Von Eschwege, mineralogista e metalurgista alemão, que esteve em Minas Gerais, no início do século XIX; arrojadita, homenagem ao professor da Escola de Minas de Ouro Preto Arrojado Lisboa; pennaita, em homenagem ao engenheiro José dos Santos Penna, diretor do ITI; geannettita, em 
Publicação do ITI

Boletim 5 - 1947

ESTADO DE MINAS GERAIS

INSTITUTO DE TECNOLOGIA INDUSTRIAL

BOLETIM 5

\section{ORIGEM DAS \\ ROCHAS ALCALINAS}

POR

DJAI.MA GUIMLARES

BELD HORLONIE

ORÁFICA OUEIROZ BREINER LTDA. 
homenagem ao engenheiro formado pela Escola de Minas de Ouro Preto, Américo René Gianetti, que foi prefeito de Belo Horizonte e secretário de Agricultura do Estado de Minas Gerais, responsável pela elaboração do primeiro plano abrangente de desenvolvimento econômico do Brasil - O Plano de Recuperação Econômica e Fomento da Produção, de 1947 -, além de ter sido industrial e líder empresarial.

$\mathrm{Na}$ década de 1930, em Minas Gerais, Djalma Guimarães deu sequência às suas pesquisas com trabalhos referenciais como são: À margem de "os satélites do diamante", publicado em 1934, pelo Departamento dos Serviços Geográfico e Geológico, da Secretaria de Agricultura do Estado de Minas Gerais (GUIMARÃES, 1934). No mesmo ano, Djalma Guimarães, em parceria com Octávio Barbosa, publicou Mapa geológico do Estado de Minas Gerais (GUIMARÃES; BARBOSA, 1934), que, juntamente com Resumo de geologia do Estado de Minas Gerais(BARBOSA, 1934), constituiu as bases para as pesquisas geológicas desenvolvidas pelo ITI.

Em 1945, Djalma Guimarães apresentou ao diretor do ITI, José Moreira dos Santos Penna, um programa de pesquisas de recursos minerais do Estado de Minas Gerais, em que estavam listados depósitos minerais que deveriam ser objeto de ações do ITI complementares ao que já estava sendo desenvolvido pelo Departamento Nacional de Pesquisa Mineral. São eles: minério de ferro; manganês; alumínio; cobre; cromo; níquel; estanho; metais para aços rápidos e especiais (vanádio, tungstênio, molibdênio, scheelita, stolzita); berílio, nióbio (columbio) e tântalo; dolomita; magnesita; calcário; fosfatos e sais potássicos; e outros refratários (GUIMARÃES, 1946, p. 1-10). Em 1946, Djalma Guimarães publicou os primeiros resultados do programa de pesquisa definido em 1945, com o título Notas preliminares sobre a jazida do Barreiro. Município de Araxá, em que se revelou a existência de importantes jazidas de fosfato de cálcio (apatita), minério fundamental para correção de solos e aumento da produtividade agrícola, sobretudo porque as jazidas de Araxá continham considerável presença de radioatividade.

Conclui-se, assim que a rocha rica em fosfato de cálcio, economicamente explorável para fertilizante, apresenta um grau de radioatividade acima do comum, o que a coloca em situação privilegiada como fonte de suprimento de matéria prima para superfosfatos. Até hoje não havia sido encontrado matéria prima desse tipo.

É sabido que solos moderadamente radioativados imprimem maior vitalidade nas plantas e crescimento mais rápido. Sobre esse fato foram feitas inúmeras experiências na França e Estados Unidos (GUIMARÃES, 1946, p. 32). 
Na introdução de seu estudo sobre a jazida de Araxá, Djalma Guimarães deu particular ênfase aos aspectos geoeconômicos da questão, chamando a atenção para o grande desafio representado pela crise da atividade agrícola:

"Urge adotar medidas no sentido de .......... a indústria agricola, pois sua deficiência acarretará maleficios muito mais graves do que se podem avaliar.

Até hoje nada foi feito em relaçâo aos mais importantes problemas ligados à conservação do solo, tais como prevenção contra erosão, aumento de rendimento das colheitas, das pastagens e reflorestamento.

Não é de hoje que a ciência demonstrou ser o fósforo elemento necessário à nutrição do homem, animais e plantas (GUIMARÃES, 1946, p. 11).

A atuação do ITI foi reconhecida nacional e internacionalmente. As análises realizadas em seus laboratórios tinham como que um selo de qualidade, como se pode ver no trecho - "E assim, exportações de minérios de vários Estados do país, mesmo do norte longínquo, eram feitos mediante análises, exigidas pelos escritórios estrangeiros, europeus ou americanos, do "Laboratório da Rua da Bahia 52 - Brasil" (INSTITUTO DE TECNOLOGIA INDUSTRIAL, 1958, p. 6).

A descoberta da jazida de Araxá é um dos marcos da geologia brasileira e da carreira de Djalma Guimarães, que detectou ali não só consideráveis reservas de minerais fosfáticos (apatita), mas também a maior reserva mundial de nióbio (pirocloro). Djalma Guimarães também foi pioneiro na detecção de minerais radioativos no Planalto de Poços de Caldas (BRANCO, 1956).

Em balanço da atuação do ITI, realizado em 1958, constatou-se que o Instituto havia publicado, até àquela data, 113 trabalhos sobre os seguintes temas: exploração de jazidas minerais; propriedades de máquinas operatrizes; aproveitamento de águas e de jazida de fertilizantes; estudos sobre desenvolvimento agrícola; energia nuclear; os minerais radioativos e sua localização e extração (OLIVEIRA, 1958, p. I-II).

O setor de Geologia e Geoquímica do ITI, organizado por Djalma Guimarães, contou com a colaboração de uma equipe de engenheiros, químicos e geólogos com reconhecimento nacional e internacional, tais como Boris Brajnikov, Iphygênio Soares Coelho, Vladimir Belezkoij, André Scheider, Wilfried Drawin, Mário C. Drumond, Antônio Barbosa Otoni, Otávio Barbosa, Manoel Teixeira da Costa e outros. O setor de Química, cujo laboratório foi inicialmente chefiado por Caio Pandiá Guimarães, atuou como complemento indispensável aos trabalhos desenvolvidos pelos setores de Geologia, Mineralogia e Petrografia. Em 1951, foi instalado 
Publicação do Instituto de Pesquisas Radioativas

Publicação $\mathrm{n}^{\circ} 5$

1956

ESCOLA DE ENGENHARIA

UNIVERSIDADE DE MINAS GERAIS

DIRETOR: PROF. MARIO WERNECK DE ALENCAR LIMA

INSTITUTO DE PESQUISAS RADIOATIVAS

DIRETOR: PROF. FRANCISCO A. MAGALHĀES GOMES

\section{PUBLICAÇÃO N. ${ }^{0} 5$}

\section{NOTAS SOBBE A GEOLOCIA E PETROGRAFIA DO PLANALTO DE POCQOS DE CALDAS MG.}

JOSE JAIME RODRIGUES BRANCO

BELO HORIZONTE

1956 
no ITI o setor de Espectografia, mediante a aquisição do espectógrafo de retícula de alto poder de resolução, que permitiu ao ITI sediar o mais complexo laboratório de espectoquímica da América do Sul. Pioneira, também, foi a atuação do ITI no desenvolvimento de pesquisas sobre radioatividade e energia nuclear:

Técnicos do Instituto já têm feito cursos de especialização nos Estados Unidos e na Europa, a fim de preparar melhor sua equipe para acompanhar, dentro do possivel, o que se realiza no gênero nos centros mais avançados do mundo. Assim, o Prof. Milton Campos, realizou um curso de Radioquimica em Chicago, enquanto o prof. Willer Florêncio estudou e trabalhou em indústrias de matérias radioativas em La Rochelle, na França.

O vulto dos trabalhos realizados por este núcleo técnico, nascido no antigo serviço de Produção Mineral e hoje representado pelo Instituto de Tecnologia Industrial, assumiu tal significação em seu remarcado progresso que veio contribuir para o estabelecimento de um novo e mais avançado nivel de atividades técnico-cientificas, nas Minas, qual seja o do recentemente criado Instituto de Pesquisas Radioativas da Escola de Engenharia da UMG. Sem dúvida alguma, esta nova e auspiciosa fase, está implantada no ambiente previamente preparado pelo labor deste grupo de pioneiros constituido pela Escola de Engenharia - Instituto de Tecnologia Industrial (INSTITUTO DE TECNOLOGIA INDUSTRIAL, 1958, p. 14-15).

Em 1937, o Serviço da Produção Mineral do Estado de Minas Gerais montou laboratório para estudo das fontes hidrominerais de Minas Gerais, trabalho este que continuou a ser feito pelo ITI, assim como o estudo químico dos solos de Minas. O Serviço de Metalurgia Física do ITI teve significativo desenvolvimento durante a gestão do engenheiro José Moreira dos Santos Penna, segundo diretor do ITI e responsável pela consolidação e expansão, tendo sido equipado para atender às demandas do setor industrial mineiro, em particular suas indústrias metalúrgicas e siderúrgicas. O laboratório de metalurgia do ITI foi um dos primeiros do país a possuir um microscópio eletrônico e um aparelho de raio-X de alta potência para radiografia de peças metálicas. O ITI manteve desde o início um laboratório de resistência de materiais e uma oficina mecânica de alta precisão equipada com aparelhagem atualizada, atendendo às demandas tanto de outros setores do Instituto, quanto de outros órgãos do Estado e do setor privado. Também significativas foram as iniciativas do ITI no desenvolvimento de tecnologia de exportação de óleos ve- 
getais para fins energéticos, como babaçu, mamona e macaúba. Tanto a indústria madeireira quanto a indústria de fibras e de tecidos foram contempladas no ITI com a instalação dos laboratórios especializados no estudo de melhorias tecnológicas para estas indústrias. Com repercussão abrangente foi a atuação do setor de metrologia do ITI, que "possui um dos melhores laboratórios de medida de alta precisão do país, podendo aferir calibres e fazer verificação de peças" (INSTITUTO DE TECNOLOGIA INDUSTRIAL, 1958, p. 20). O ITI abrigou, também, uma fábrica de sulfona, que depois foi transferida para o Departamento de Lepra da Secretaria de Saúde.

Durante toda a década de 1950, o ITI funcionou na rua da Bahia 52, no antigo Instituto de Química da Escola de Engenharia da UFMG, na expectativa de construção de nova sede, mais ampla e especialmente equipada para atender às especificidades do órgão. Quando ficaram prontas as novas instalações, na Cidade Industrial, o ITI já se encontrava em crise irreversível e as instalaçōes foram transferidas para a Companhia Energética de Minas Gerais (Cemig).

O documento já citado aqui mais de uma vez - Histórico e atuação do ITI no desenvolvimento técnico-cientifico em Minas Gerais - é um balanço equilibrado e particularmente atual sobre certas características estruturais do desenvolvimento científico e tecnológico nacional, seja na explicitação de seus efetivos avanços e conquistas, seja na reiteração de seus impasses, descontinuidades, assimetrias e precariedades.

Com efeito, o recorrente, e justo, diagnóstico sobre a imaturidade e incompletude do Sistema Nacional de Inovação no Brasil não é apenas, nem especialmente, o resultado do atraso do país em criar instituições científicas e tecnológicas. De fato, em muitos e importantes aspectos e setores, o país esteve, relativamente, sintonizado ao que se fazia de mais avançado no mundo, e mesmo teve instituições com certo protagonismo em suas áreas, como, por exemplo, o Instituto Agronômico de Campinas, criado em 1887, a Escola de Minas de Ouro Preto, fundada em 1876, e o Instituto de Manguinhos, criado em 1899.

No seu tempo e em sua área de atuação, o ITI teve desempenho exemplar. A Instituição, ao destacar suas grandes contribuiçôes, listou três que, efetivamente, superaram a mediania:

- a descoberta de sete novos minerais - além dos quatro descobertos por Djalma Guimarães, já mencionados, têm-se outros três: a djalmita, nome dado em homenagem a Djalma Guimarães, mineral radioativo contendo urânio, tântalo e nióbio, descoberto por Caio Pandiá Guimarães; a ribeirita, nome dado em homenagem ao físico brasileiro Joaquim Costa Ribeiro, 
descoberto por Willer Florêncio; e a alvarolita, nome dado em homenagem ao Almirante Álvaro Alberto, fundador do CNPq, um tantalato de manganês, descoberto por Willer Florêncio;

- os estudos sobre minerais radioativos a partir dos trabalhos de Djalma Guimarães, Willer Florêncio, Caio Pandiá Guimarães e Celso de Castro;

- as pesquisas sobre o tempo geológico da terra: "Pesquisas concernentes às idades das rochas e minerais componentes de diversas formaçôes geológicas [...] visando determinação de dados mais exatos com referência à idade da terra e de seus períodos geológicos. Estes estudos são baseados no conhecimento das leis de transformação dos elementos radioativos." (INSTITUTO DE TECNOLOGIA INDUSTRIAL, 1958, p. 27).

\section{Instituto de Quimica da Escola de Engenharia da UFMG onde funcionou o ITI. "Rua da Bahia, 52 - Brasil"}

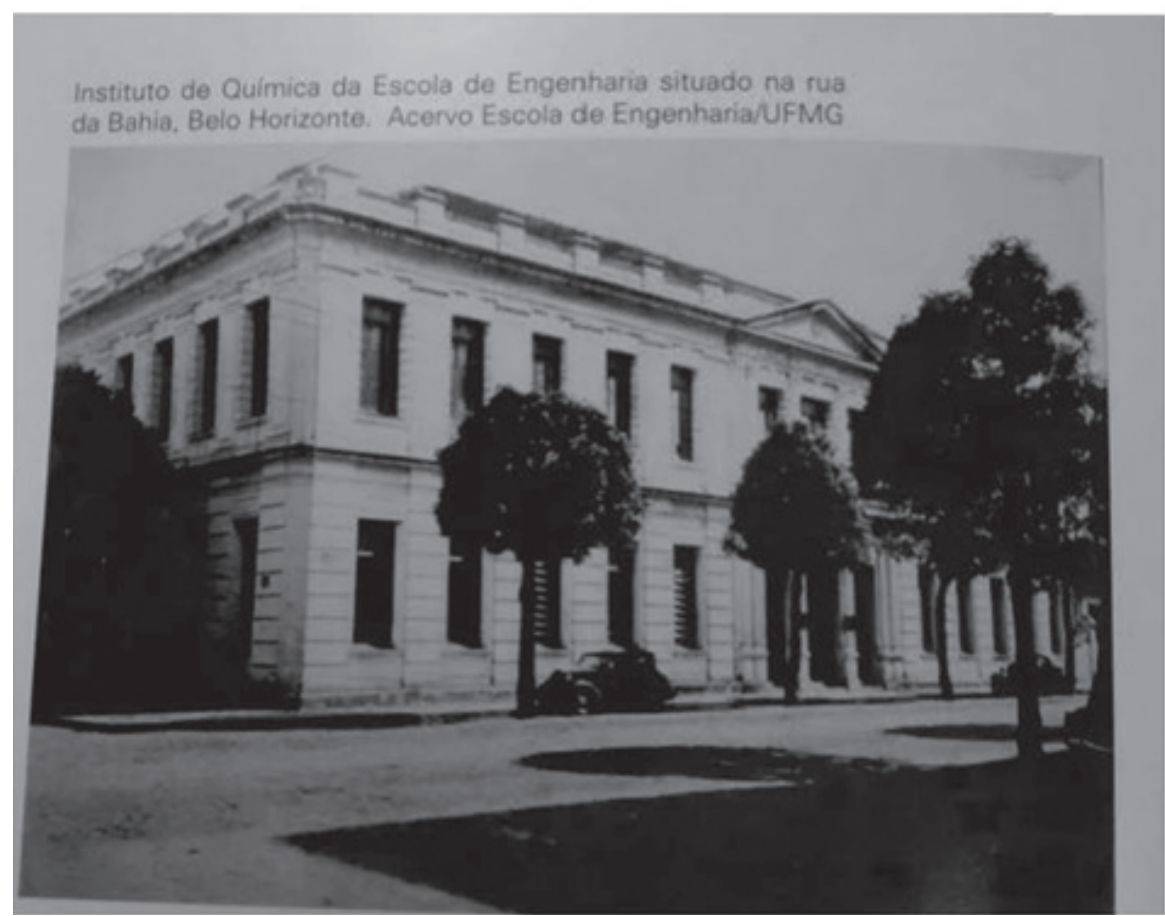


A crise que se abateu sobre o ITI, a partir do final dos anos 1950, é de difícil explicação porque tem origem num paradoxo. É fácil explicar uma crise, a extinção de uma instituição, de um ser vivo, de uma sociedade, de um processo como resultado de um fracasso, de um colapso, de entropia radical. Muito difícil, no entanto, é explicar uma crise, a extinção de uma instituição, no caso, quando esta foi bem-sucedida, e antes que atingisse seus objetivos, se isso fizer sentido para instituiçōes que visam o desenvolvimento científico e tecnológico que é, por certo, rigorosamente, o objetivo permanente e inesgotável.

Acontece que o ITI foi abandonado à morte luta, por inanição, que se prolongou até 1972, quando foi criado o Cetec, inicialmente ligado à Fundação João Pinheiro: "Sucedendo, na Administração Estadual, ao antigo Instituto de Tecnologia Industrial, de respeitável tradição, o CETEC estabeleceu como política não substituir a capacidade de pesquisa aplicada existente em Minas, mas, ao contrário, cooperar para ativá-la, utilizando-a mediante contratos ou subcontratos de pesquisa" (CINTRA, 1979, p. 18).

Com efeito, a história do ITI tem algo de desconcertante, de paradoxal, que, por outro lado, é bastante conhecido entre nós, constituindo-se, de fato, um fenômeno recorrente do Sistema Nacional de Inovação no Brasil, que é sua crônica, até aqui, incapacidade de consolidar e expandir algumas de suas experiências mais exitosas, pela intercorrência de inépcia e irresponsabilidade política e institucional.

\section{Referências bibliográficas}

ÁVILA, Cristina. Estudo crítico. In: GRAVATÁ, Hélio. Resgate bibliográfico de Minas Gerais. Belo Horizonte: Fundação João Pinheiro, 1998.

BARBOSA, Octávio. Resumo de geologia do Estado de Minas Gerais. Belo Horizonte: Serviço Geológico, Departamento dos Serviços Geográfico e Geológico, Secretaria de Agricultura do Estado de Minas Gerais, 1934 (Boletim 3).

BRANCO, José Jaime Rodrigues. Notas sobre a geologia e a petrografia do Planalto de Poços de Caldas. MG. Belo Horizonte: Instituto de Pesquisas Radioativas, Escola de Engenharia, Universidade Federal de Minas Gerais, 1956 (Publicação n. 5).

CARVALHO, Antônio Gontijo de. Calógeras. São Paulo: Companhia Editora Nacional, 1935.

CARVALHO, José Murilo de. A Escola de Minas de Ouro Preto. O peso da glória. Rio de Janeiro: Finep, 1978. 
CINTRA, Antônio Octávio (Org.). Fundação João Pinheiro. Notícia histórica, áreas de atuação, trabalhos realizados. Belo Horizonte: FJP/SEPCG, 1979.

DINIZ, Clélio Campolina. Lucas Lopes. O visionário do desenvolvimento. Revista do Arquivo Público Mineiro, ano XLIV, n. 2, jul./dez. 2008.

GUIMARÃES, Anchieta. Secretários de Agricultura de 1933 a 1988: esboço-administrativo. Belo Horizonte: Seapa, v. I, 1990a.

Secretários de Agricultura de 1933 a 1988: esboço-administrativo. Belo Horizonte: Seapa, v. II, $1990 \mathrm{~b}$

GUIMARĀES, Djalma. Contribuição à petrographia do Brasil. Rio de Janeiro: Serviço Geológico e Mineralógico do Brasil, Ministério da Agricultura, Indústria e Comércio, Imprensa Nacional, 1924 (Boletim n. 6).

À margem de "os satélites do diamante". Belo Horizonte: Serviço Geológico, Departamento dos Serviços Geográficos e Geológicos, Secretaria da Agricultura do Estado de Minas Gerais, Oficinas Gráficas da Estatística, 1934.

Nota preliminar sobre a jazida do Barreiro, município de Araxá. Belo Horizonte: Instituto de Tecnologia Industrial, Veloso \& Cia. Ltda., 1946.

GUIMARÃES, Djalma; BARBOSA, Octávio. Mapa geológico do Estado de Minas Gerais. Belo Horizonte: Serviço Geológico do Estado de Minas Gerais, Secretaria de Agricultura, 1934. INSTITUTO DE TECNOLOGIA INDUSTRIAL. Histórico e atuação do ITI no desenvolvimento técnico-científico em Minas Gerais. Belo Horizonte: ITI, 1958.

OLIVEIRA, José Marcelino de. Introdução. In: INSTITUTO DE TECNOLOGIA INDUSTRIAL. Histórico e atuação do ITI no desenvolvimento técnico-cientifico em Minas Gerais. Belo Horizonte: ITI, 1958.

SCHWARTZMAN, Simon. Um espaço para a ciência. A formação da comunidade científica no Brasil. 2. ed. Campinas: Editora da Unicamp, 2015. 


\section{Publicação do ITI}

1946 - 1958

\section{APENDICE A \\ Publicacōes eflcla1s}

de

Inst1tute de Tecnolorda Industrial de Bstade de U1nas cera1s

1 - GUTMARIBS, D.

Contribu1cäo ì rênese dos magmas tolefticos 1946 - Boletim ne 1

* Bste assir como os boletins (2) e (4) tratam da gênese de cer tgs tipos de rochas 1 gneas e constituem trabalho de pesquisa sobre problemas fundamentais de geologia. Todos alcançaram sepercuçào Internacional.

2 - GUIMART̃ES, D.

Bnstenit1zacão 을 zoneamento dos plardoclásios 1946 - Bolet $1 \mathrm{~m} n \mathrm{n} 2$

3 - PLORÊNCIO, $\mathrm{z}_{2}$ $\frac{\text { Mod1r1casấo } 1 \text { ntroduz1da ne processe de schoeller }}{1946 \text { - Avulso } n^{2} 1 \text { (no prelo) }}$

4 - GUIMARZES, D.

Orizea das rochas alcalinas

5 - GUTMARTES, D.

Nota pre 1 iminar sôbre a 1az1da de Barre1ro, Araxá, Linas cerats.

$$
1947 \text { - Avulso ne } 2
$$

- Nota prévía sôbre a descoberta, pelo I.T.I., da grande jazida de apatita de Araxá (100 milhöes de toneladas de rocha fosfat1ca).

6 - PLORGMCIO, w.

$\frac{\text { Uraninita }}{1948-\frac{\text { Be }}{\text { Avulso } 11}}$

7 - GUTYARTES, CAIO PANDÍ́

Recorrencia de mineral1zacão elm depósitos caledonianos, d.18lingt ta 2 calogerasita.

1948 - Boletsin no

8 - GUTMARJES, D.

The zirconium ore deposits of the Pocos de Caldas plateau, Braz11, and z 1 rcon 1 um Gooches 1 stry. 1948 - Boletim n' 6

- Trabalho que estabelece a gênese dos depósitos z1rconfferos de Poços de Caldas. 
9 - BRAJNIKOV, B. e GUTUARÃES, D.

Enstenitization in the Palisade s1ll diabase and 1tg consequenceg.

1948 - Boletim $n^{2} 7$

10 - FLOREHCIO, a.

Observacöes sôbre a 1onizacīo do ar durante e eclínse to-

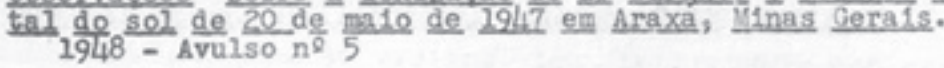

11 - CASTRo, C. e FLORENCIO, ,

Aquas, terma1s de Itaý

12 - FLORBACIO, W.

Keva fente rad1oat1va de Bacre1ro, Axaxá, M1nas Gera1s.

Agua da "Bomba". 1948 - Avulso ne 7

13 - FLORENCIO, 1. $\frac{\text { Harcha analftea dos minerals do srupo da betafita }}{1948 \text { - Avulso }}$

14 - PLORANCIO, w.

Cronogeoleg1a dos pegmat1tos braslleiros

1949 - Avulso ne?

- Trata-se de trabalho p1one1ro sôbre determ1naçãa de 1dgde dos pegmat1tos bras1le1ros, usando-se a relação urân1o e tor1o pa ra chumbo.

15 - BRAJIIKOV, B.

Les rapperts numèriques et volumétriques de l'oxyrène dans Les mineraux et les roches. $1949-$ Boletin ${ }^{2} \frac{10}{8}$

16 - GUIMARÄES, D.

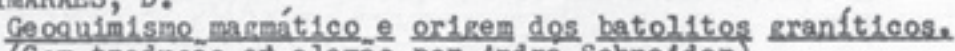

(Com traduçâo ed alemâo por And re schne1der)

1949 - Bolet1m ne 9

* Nova expos1ção sôbre a teor1a đa gran1t1zação apresentada pela prine1ra vez no Congresso Internacional de Geologla de Ber 11m em 1936.

18 - MENICUCCI SOBR INHO, L.

Estudo quimico da apat1ta de Araxá, Minas Gera1s 1949 - Avulso ne 10

19 - GUIMARÃES, D.

$\frac{\text { Bncore à propos de la génèse des orthopyroxènes }}{1952 \text { - Avulso } \mathrm{n}^{2} \text { II }}$ 
20 - PEIXoTo, F2 nerals 1 1ras.

$1952^{2}$ - Avulso $n e 12$

21 - BRAJNIKOV, B.

Carte des directions de c1salllements dans le Brés1l Cen-

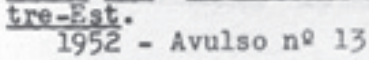

22 - FLORBACIO, W.

$\frac{\text { Linerals }}{1952}$ - de urân10 $\frac{\text { tór1e }}{\text { Bet1m }}$

* Catalogação de 196 minera1s de urân1o e tór1o; 77 minera1s que contem urânio e tọr1o em pequena percentagem; e 36 quę pe dem conter uranio e torio como inpurezas. Cada minerai é âpresentado com uma descriçáo detalhada de sua composiçáo quimica, propriedades fisicas e ocorrencias.

23 - LEMZ, ע. F.

Prática de análise de rocha

$1952-B o l e t 1 m$ n 12

24 - PIRES E ALUUQUERQUE, 0. A. L.

Fresadoras

1952 - Boletin ne 13

25 - FLORBNCIO, w.

Rad1oatividade e fert111zacãe 1953 - Avulso $13 a$

26 - COELHо, I. S.

Consideracōes sôbre os depósitos mankanesferes de üinas Cera1s: 1953 - Avulso ne ll

* Centem uma avaliação da reserva conhecida de minério de manga nes em M1nas Gegrais. Tem alcançado grande repercuçäo nos nos sos me1os economicos.

27 - ILCHËNKO, V. e GUIRARÃES, D.

Sobre a posslbilidade de ut1ulzacĩo agricola dos stenitos nefelintces de planalto de Poges de caldas, Minas Gerais. 1953 - Avuiso ne 15

28 - GUIMARZ̃ES, D.

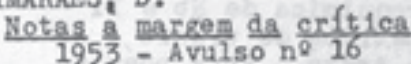

29 - GUTHARAES, D.

Aga1n on the genes1s of the orthopyroxenes 
30 - BEIBZKIJ, X. - GUTMARÃ̈S, D. e COBIHO, I. S.

Netas sobre alrueas lazidas de minérle de manganês e ferre de Xinas Gersis.

1953 - Boletin $n^{2} 15$

31 - GUTMARZES, D.

Areas reolostcally faverable to occurence of thor1um and uran1um in Braz11.

32 - OLIVEIRA, J. $x$. DE,

Desaren de yanád10 Dele tan1ne. Análise da vanadin1ta, desclo1sita e 11 liemita. 1954 - Avulso ne 18

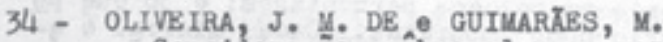

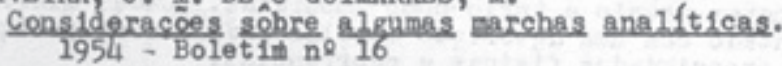

35 - CASTRO, C. \& OLIVEIRA, J. y. DE

Alrumas águas minera1s de Bstade de Ge1ás 1954 - Bolet1m n² 17

36 - CASTRO, C.

0 sole: sua v1da e sua morte

37 - MORABS, L. J. DE E GUTMARZ̃ES, D.

Im minerai rare dos colaitos de Serrete, Cascata, mun1efDle de Aruas da Prata, Sae Paute. 1955 - Avulso n 19

38 - ILCHENKO, $\mathrm{v}$.

Yodiflcacãe de n1vel enerrét 1ce estrutural de alsuns scistals com a vartacae do numere de ceordenacae do cation. 1955 - Aviviso ne 20 .

39 - GUMARTES, D.

Contributcie a estude des tures yulcântces da Mata da Corda. 1955 - Bolet1m ne 18

40 - DURRA, C. V. e DUTRA, C.

Trabaine analftice sôbre a pireclerita de Araxí e Tapira, Hinas Gerais.

I Determinação espectroquínica de 짜 e T1

II Detxerminaçâo fluorometrica de uranio,

III Uranio na apat1ta de Araxa - Nota prévia

1956, Bolet1m ne 20

- Contem a descrição detalhada dos métodos_espectroqufmicos origina1s estabelec1dos para a detęrminação de H1ob1o - T1 tanio nas rochas do Barreiro, Araxá, tambéa sôbre a flue rómetria do uranio no mesmu material. A terceira parte 
uma notfola prévia sôbre as pritselras determinaçōes de urî n1o na apatita e cuja reserva é hoje aval1ada en 30.000 toneladas de $\mathrm{U}_{3} \mathrm{O}_{8}$.

41 - GIIIARR̃ BS, D.

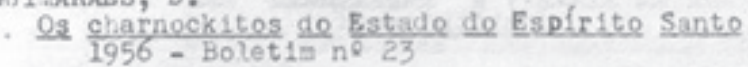

42 - NEVES, A. T2

Introducia ao estude anatônlce das madelras

1957 - Boletin ne 2i

43 - PEREIRA, 0. V.
$\frac{\text { Cenese das águss }}{1958} \frac{\text { subterrâneas }}{n^{2} 22 \text { (No pre 10) }}$ 
APÊNDICE B

\section{Publicacões, referentes a trabalhos executades per runclonarios técnicos de I.T.Te, nes dabe= ratortos do I.T.I., e pubilcades per eutras Inst 1tulees:}

44 - COELHO, I. S.

Ocorrênc1a de manganês na Colôn1a Yarzem Grande, Hun1efole

de Bele Hordzonte.

Iineraçao e Metalurg1a, 1941 (Vol. V, ne 30)

45 - FLOBÊNCI0, W. E MENICOCCI SOBRINHO, L.

Aguas minera1s de Bstade de Minas Academia Nacional de Farmacia, 1941 (Ana1s)

46 - GUIMARZ̃ES, C. P.

Recorrencia de mineral1zacão em depós1tos galedon1anes Acad. Bras. de C1encias, 1942 (Vol. XIX, $n^{2} 3$ ).

47 - GODOY, x. P. DB

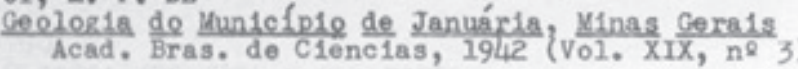

48 - COBLHO, I. S.

Ocerrencla de Cass1ter1ta em_São João del Re1, Minas Ge ra1s. Yineração Metalurg1a, 1943 (ne 36)

49 - FLORENCIO, W. e CASTRO, C. DE

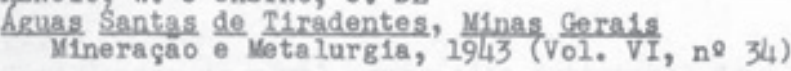

50 - FLORENCIO, W.

Aguas minerals Serv. Prod. Mineral da Sec. da Agr. de Minas Cerals (Vol. I).

51 - FLORÊNCIO, W.

Rad1oat17idade da conte de ágma $\mathrm{D}$. Pedre, Caxambú, u1nas

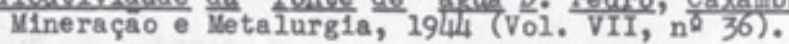

52 - GUIMARZES, D. A COELHO, I. S.

Nota preliminar sôbre as laz1das de minérle tungstên1ce em Sumidoure, Martans, Yinas Gera1s. D.R.P.u., R10 19 44 (Avulso ne 60 )

53 - guTMARÃBs, D. e CDBLHO, I. S.

Bauxita de Yorre do Cruzeire, oure Preto, M1nas Gera1s. D.N.P.Y. - R10 1945 (Boletin no 67) 
54 - GUTMARXES, D,

Jazidas minersis de or1rem magmít1ca

10 Congresso Pan-Americano de Bngenhar1a de winas e Ges $\log 1 \mathrm{a}-1946$ (Ana1s)

55 - GUTWARZSS, D.

Hoticlas de 18 Concresse Pan-Anercane de Bngenhacla de

kinas \& geoloria.

Lrquiteture Engenhar1a - 1946 ( $n$ e 3)

56 - GUTMARLES, D. O COELHO, I. S.

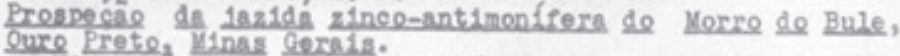
Ninaraço Uetalurg1a, 1946 (ne 61)

57 - GUTMAREES, D.

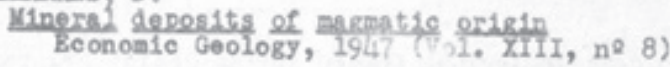

58 - FLORBNCIO, W. - CASTRO, C. de

Uraninita Minas Gera1s Acad. Bras, de Clenc1as, 1967 (Ana1s, Tomo XV)

59 - GODOY, M. P. e cos

Recurses minerais de Bstide de vinas Geras Sec. Agricultura de Minas Gera1s, 1947

60 - cosia, I. S.

Turmalina cibrese de Uins de cruzedro Íne raçåo Wotalurgis, 1948 (vol. XIII, n2 73)

61 - RIBEIRO, J, $\mathrm{J}_{2}$. E FLORÊNCIO, W.

Aplicacas das pedidas de teôr $10 \hat{n} 1 c 0$ de ar ì pesau1za e ì prespeccae de minera1s rediegtivos.

Acad. Bras. de C1encias, 1948 (Tomo XX, ne 4)

62 - GUTMARTES, D. - OTTOYI, A. B. E FLOBENCIO, W.

Obserracees zeoloricas, Detrerríficas 2 minerulóricas en Loeda, vinas cora1s. Acad. Bras. de Clêne1as, 1948 (Tomo XX, $n \in$ 4)

63 - FLORENCIO, พ.

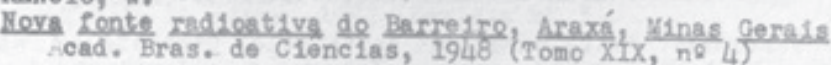

64 - GUTUARABS, D.

Age deternination of quartz veing and pegmatites in Brazil Bconom1c Ceology, 1948 (vol. XLIII, ne 2)

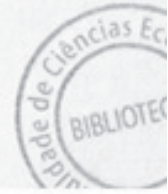


65 - GUTILARÃEs, D.

La_renese des orthopyroxènes Sncleté Ceologique de France, 1948 (Tome XVIII, Bul.)

66 - FLORÊNCIO, พ.

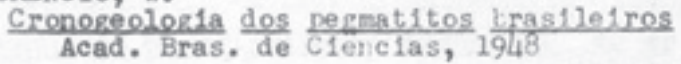

67 - GUIMART̃ES, D. e PLORENCIO, $x$.

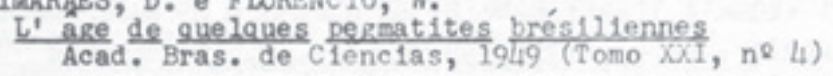

68 - CASTRO, C. e PLORERCIO, \%.

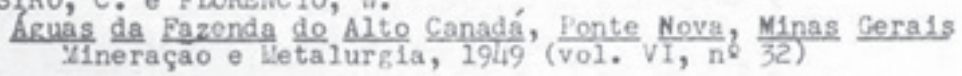

69 - GUIMARAES, D.

Chronelogle géolerique fondée sur la désintegration aton1que des mineraux radioactics. Soc1ete Ceologique de France, 1949 (Bul. Tone XIX)

70 - CASTRO, C. e FLORENCIO w

Acuas minerals de Tap1ra, Sacrapento, M1nas Gerais Mineraçao e Wetalurg1a, 1950 (Vol. VII1, n.43)

71 - GUTMARTES, D.

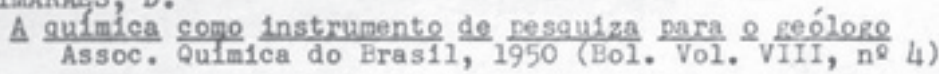

72 - GUMLART̈BS, D.

o est1 lo da evolucão geodinâm1ca do escudo bras1le1ro e sua incluenc1a nos processos metalozeneticos. Assoc. Bras. de Metais, 1950 (Bol. vol. VI, ne 20)

73- GUIMARД̈BS, D.

A lazida de dialmalta de Volta Grande, Rle das Mortes, u1nas Gera1s. Acad. Bras. de C1ênc1as, 1950 (Tomo XXII, ne 1)

74 - TAVORA, E. e FEIXOTO, F.

Latifee constants of tan1olite from Mecelana, Fortaleza, Ceara. Acad. Bras. de Clências, 1951 (Vol. XXIII, ne 4).

* Pesquiza cristalográfica fe1ta pelo Gabinete de Petrografla do D.N.P.u. - R1o, com a colaboração do Inst1tuto de Tecnologla Industrial - Belo Horizonte.

75 - FLORENCIO, W. - BRAJNIKOV, B. - JUNQUE IRA, G. S. DE 0.e GUIMA RÃES, D.

Leteor1to "Morro Cavado", Conce1cão da Aparec1da, M.G. Acad. Bras. de C1enclas, 1951 (Ana1s.) 
76 - GUTMARAES, D.

$\frac{\text { Arqu1-Brasil e sua evolucão Reolózlca }}{\text { D.P.P.I. }}$

77 - GUTUARRES, $\mathrm{x}$.

A constitu1cãe cristalina dos alumo-s111catos e sua anál1.

se termica.

Ass. Qufm1ea do Bras11, 1951 (Bol. Vol. IX, ne 4)

78 - FLRÊNCIO, W.

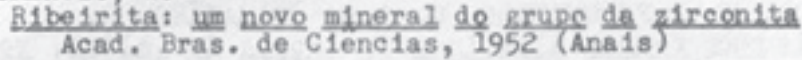

79 - GUIMARAES, D.

A proposito de processo de enatentt1zacäe

Acad. Bras. de Clenc1as, 1952 (Vol. XXIV, $\mathrm{n}^{2}$ 1)

80 - PLORÊNCIO, W.

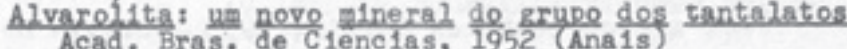

81 - GUTUARZZS, D.

$\Delta$ proposito de rênese dos ortoptroxênles

Acad. Bras. de Clenc1as, 1952 (vol. XxIV, $\mathrm{n}^{2} 2$ )

82 - GU TMARK̈BS, D.

D1retrizes clentfelcas adotadas na pesquiza de depósitos grantferes.

83 - BHERING, J. B. O GU IMARÃES, D.

Recuperacáe de Vale de säe Franc1sco

Bscola de unas, 1952 - (Rev1sta, Ma10)

* Notável estudo, precursor de relevantes medidas econôm1cas e socia1s, ea que colabornu o Chefe da D1visáo de Geologia e M1 nes do I.T.I.

84 - GUTMARAES, D.

Iazidas minera1s de er1rem magnátice

Escola de Minas, 1952 (Revista $n^{2}$ 6)

85 - GUTMARTES, D.

Observacöes sôbre a reodinâmica de escude bras1le1re Bscola de Yinas, 1952 (Rev1sta $n^{2} 3$ ).

86 - PEIXOTO, P. GUIMARIBS, D.

Problemas de sronereoleria

Acad. Bras. de Clenc1as, 1952 (Vo1. XXIV, ne 3) 
87 - BRAJNIKOV, B. E GULMARÃES, D.

Xetes sur le probleme chronozéologique Acad. Bras, de C1encias, 1952 (vol. XXIV, ne 3)

88 - PEIXOTO, F. G GUMARZBS, D.

Problenas de cronogeolozia

Inst1tuto de Pesquisas Radioativas, Belo Horizonte, 1953 (Bolet1n ne 1)

89 - CAMPOS, M. - FILGUEREDO D. G. - GUIMARAES, D.

Alrumas rochas alcalinas de Poces de Caldas, relacionadas com as 1azldas de caldas 1 to uranlfero

Inst. Pesquisas Rad1oativas, Belo Hor1zonte, 1953 (Bo letim $n$ 2).

90 - ILCHE्ENKO, V. A GUIMARZES, D.

Sôbre as pess1b111dades de emprege do cola1to de Pocos de Caldas como fert1lizante potassico. Sec. Agr1e. de Minas Gera1s, 1953 (Bolet1m ne 5 \& 6)

91 - PERE IRA, O. V. O CASTRO, C. DE

$\Delta$ captacãe da conte mineral Santa clara, Guarapuava, Parana. Publicação do Govêrno do Paraná, 1953.

92 - ILCHENKO, V. e GUILARÃES, D.

A ut1lizacão come fert1ilzante potássice dos folaltos de Pocos de Caldas. Winas Kagazine, 1953 (We 5).

93 - DUTRA, C. V. E MURATA, K. J.

Spectrochenical determination of thorium in monazite, by the poeder-d,c. arc techn1que. spectroch1alca Acta - Londres, 1954 (vol. VI)

94 - ILCHBNKO, V. e GUIMARÄBS, D.

Rochas cem pseudo-leuc1ta ou ep1-leuc1ta de Pocos de Caldas. Sec. Agr1c, de Minas Gera1s, 1954 (Bolet1m 1 \& 2)

95 - IICHENKO, V. E GUINARZES, D.

Apat1ta do Barre1re, Axaxá, Minas Gera1s

Sec. Agr1cultura de Minas Gera1s,1954 (Bolet1ns 7 a 12)

96 - PRREIRi, 0. V.

Gerajs.

Pubilcação da Prefe1tura de Santos Dumont, 1955

97 - ILCHENKO, V. e GUIMARẌES, D.

Decomposicâo de rochas alcalinas do platean de Pocos de

Caldas.

Sec. Agr1c. de Winas Gera1s, 1955 
98 - DURRA, C. V.

Determinacão esnectroquimica de ma1ores e menores const 1tilintes, er pequenas amostras de minera1s - Analise cemple ta de lamprofi11ta e end1a11ta. (Bol. ne 4 ).

99 - NEVES, A. T.

o. cálclo cont1de nas made1ras exercerá 1nfluênc1a cono fator de preservacáo?

Sec. Agric, de Minas Gera1s, 1956 (Boletins 1 \& 2)

100 - GUIUARÃES, D.

Rochas torfferas de onca, sul de São Joãe del Re1, Minas

Gerais.

Engenhar1a, Mineração e Ketalurg1a, 1956

101 - GUTMARZ̃ES, D.

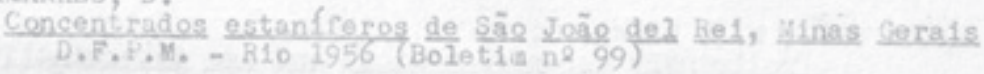

102 - BEIBZKIJ W, e GUTMARÄES, D,

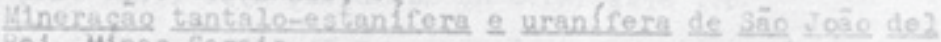

Bei, Minns Gemais

B.F.P.K., K10 1956 (Bolet1m ne 99)

103 - PEIELKIJ, W. E GUIKARABS; D.

The stane-tantalo-ucaniferous depesits and occurrences in the recion of $\frac{50}{10 a 0}$ del Hei, , inas Cera1s, Hrezil.

(Froceedings of the International

Conference on the peacerul uses of atomic energy - Geology of Uranium an Tho Mum - vol. VI).

1044 - GUIZARÃES, D,

Tratamente do granito estanffere de Volta Grande e Germinal, D1strite de Nazareno, Sae Joae del Res, Minas Gera1s. D.P.P.W., R10 1956 (Boletim ñ 99)

105 - GUIZARAES, D. Areas geologlcally favorable to eccurrence of thorlum and Geneve, Su1ssa - 1956

(Proceedings of the International Conference on the peaceful uses of atomic energy - Geology of Urantum and Thorluas. Vol. VI).

106 - VIVACqUA, A.

Bxploltation rationne110 đu babassú au Brós11

oleagineux - Paris, 1957 (n ii) 


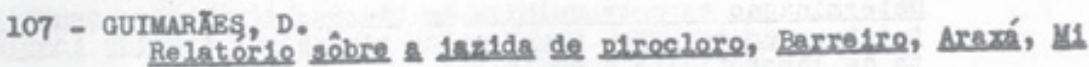

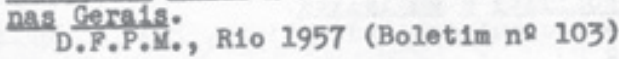

*Relatór1o final dos trabalhos executados pelo I.T.I. sôbre a descoberta a a gubagem da jaz1da de p1rocloro ( $\mathrm{Nb}, \mathrm{T}$. Raras, Th e U) de Araxa.

Resultado: 4.643.000 tons. métricas de óxido de $N 10 ́ b 10$ (a ma or Jaz1da de Nb conhec1da na atualíndade), 130.000 tons, de oxido de ţór10 e 84.000 tons. de uran1o, todos economicamente aprove1táve1s.

100 - GUTMARZES, D.

Ocorrencia de um mineral rare ne permat1to de Germinal, D1strito de Nazareno, Sao Joae del Re1, Minas Geraise Bscola de Minas, 1957 (vol. XXI, $\frac{n^{2}}{2}$ ).

109 - HBRZ, N. E DUTRA, C. V.

Breliminary espectrochemical and age determination results on some granitic recks of the "quadrilitere ferrffere", $\frac{M 1}{11}$

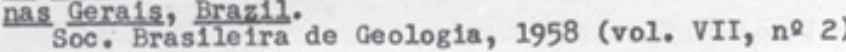

Trabalho em cooperação com o Un1ted States Geological Survey

110 - GUIMARABS, D.

Notas a margem de "일 paláde e a platina ne Bras1l", de $\mathrm{E}$. Hussak.: Soc. Bras1le1ra de Geolog1a, 1958 (vol. VII, ne 2)

111 - GUTMARIES, D. \& BBLBZKIJ, w.

Relatór10 sôbre uma ocorrênc1a singular de plat1na, geo-

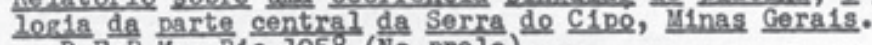
D.F.P.M., R10 1958 (No prelo).

112 - GUMMARZ̈ES, D:

Meteorlto de Córrege de Areado, Patos, Minas Gera1s Bol. da Soc. Bras1le1ra de Geologla (Vol. VII, ne 2) 1958 113 - MURATA, K. J. - DUTRA, C. V. -TE IXTEIRA DA COSTA, M. O BRANCO,

Composition of pegmatitic monazites of eastern Minas Gera1s, Braz11. Geoch1m1ca et Cosmoch1m1ca Acta - Londres, 1958 (No pre 10).

* Traba tho executado na secção de Espectroqufmica do I.T.I. em colaboração com o geoqulmico K. J. Jurata do U. S. Geological Survey.

* Notas do I.T.I. 
